\title{
Alzheimer's Disease versus Dementia with Lewy Bodies: Cerebral Metabolic Distinction with Autopsy Confirmation
}

\author{
Satoshi Minoshima, MD, PhD, ${ }^{1}$ Norman L. Foster, MD, ${ }^{2}$ Anders A. F. Sima, MD, PhD, ${ }^{1,4}$ \\ Kirk A. Frey, MD, PhD, ${ }^{1}$ Roger L. Albin, MD, ${ }^{2,3}$ and David E. Kuhl, $\mathrm{MD}^{1}$
}

\begin{abstract}
Seeking antemortem markers to distinguish Dementia with Lewy bodies (DLB) and Alzheimer's disease (AD), we examined brain glucose metabolism of DLB and AD. Eleven DLB patients (7 Lewy body variant of AD [LBVAD] and 4 pure diffuse Lewy body disease [DLBD]) who had antemortem position emission tomography imaging and autopsy confirmation were compared to 10 autopsy-confirmed pure $\mathrm{AD}$ patients. In addition, 53 patients with clinically-diagnosed probable AD, 13 of whom later fulfilled clinical diagnoses of DLB, were examined. Autopsy-confirmed AD and DLB patients showed significant metabolic reductions involving parietotemporal association, posterior cingulate, and frontal association cortices. Only DLB patients showed significant metabolic reductions in the occipital cortex, particularly in the primary visual cortex (LBVAD $-23 \%$ and DLBD $-29 \%$ vs $A D-8 \%$ ), which distinguished DLB versus $A D$ with 90\% sensitivity and $80 \%$ specificity. Multivariate analysis revealed that occipital metabolic changes in DLB were independent from those in the adjacent parietotemporal cortices. Analysis of clinically diagnosed probable AD patients showed a significantly higher frequency of primary visual metabolic reduction among patients who fulfilled later clinical criteria for DLB. In these patients, occipital hypometabolism preceded some clinical features of DLB. Occipital hypometabolism is a potential antemortem marker to distinguish DLB versus AD.
\end{abstract}

Lewy bodies are associated historically with Parkinson's disease (PD) and are found in brainstem nuclei such as the substantia nigra and locus ceruleus. ${ }^{1}$ Similar inclusion bodies have been identified in the cortices of demented patients. ${ }^{2,3}$ With the development of immunocytochemical staining, ${ }^{4,5}$ there is increasing recognition of cortical Lewy bodies in dementia patients. According to a recent report, dementia with Lewy bodies (DLB) is considered to be the second most common cause of neurodegenerative dementing disorders following Alzheimer's disease (AD). ${ }^{6}$ Distinction between these diseases becomes important from the viewpoint of pharmacological treatment and outcome evaluation. There are suggestions of differential responses to cholinesterase inhibitor treatment ${ }^{7}$ and prognosis between the two diseases. ${ }^{8}$ Adverse responses to certain drug treatments, such as severe and potentially fatal neuroleptic sensitivity, are reported to occur more often in DLB. ' Antemortem differentiation will permit better prospective selection of more uniform pure $\mathrm{AD}$ or DLB patient populations for clinical and research in- vestigations and pharmacological trials. There are consensus reports on the clinical and pathologic diagnostic criteria for $\mathrm{DLB},{ }^{6}$ but the accuracy of the clinical diagnostic criteria has been debated. ${ }^{10,11}$ There are ongoing efforts to establish accurate and objective methods for antemortem differential diagnosis of DLB.

Albin and colleagues reported an occipital metabolic reduction with autopsy-confirmed Lewy body disease using positron emission tomography (PET) ${ }^{12}$ Occipital metabolic reduction was observed also in clinicallydiagnosed PD with dementia. ${ }^{13}$ These findings have been confirmed independently. ${ }^{14,15}$ In contrast, investigators have observed relatively preserved occipital metabolism with AD. ${ }^{16,17}$ These two observations suggest that occipital metabolic reduction may be a metabolic signature specific to DLB and can be used to distinguish the two diseases antemortem. To test this hypothesis, we compared cerebral metabolic patterns of autopsy-confirmed patients with DLB and pure AD who had antemortem PET imaging. To extend the analysis, we also examined occipital metabolism in a
From the Departments of ${ }^{1}$ Internal Medicine and ${ }^{2}$ Neurology, University of Michigan Medical School, Geriatrics Research, Education, and Clinical Center, ${ }^{3}$ VAMC, Ann Arbor, MI; and ${ }^{4}$ Departments of Pathology and Neurology, Wayne State University School of Medicine, Detroit, MI.

Received Apr 5, 2000, and in revised form Mar 12 and Apr 25, 2001. Accepted for publication Apr 25, 2001.
Address correspondence to Dr Minoshima, Department of Radiology, University of Washington, Health Sciences Building NW040J, 1959 North East Pacific Street, Box 356004, Seattle, WA 981956004. E-mail: minoshim@u.washington.edu 
large number of clinically-diagnosed $\mathrm{AD}$, some of whom later fulfilled clinical diagnostic criteria for DLB.

\section{Patients and Methods}

\section{Patients}

In the first part of the study, patients with pure $\mathrm{AD}$ or $\mathrm{DLB}$ were identified between 1990 and 1996 from an autopsy database established at the Michigan Alzheimer's Disease Research Center. Postmortem diagnosis of $\mathrm{AD}$ was made according to the age-specific quantitative criteria established by Khachaturian. ${ }^{18}$ For DLB diagnosis, at least three Lewy bodies per $\times 20$ field had to be present in four fields on ubiquitin-stained sections in three of the four most commonly affected areas, ${ }^{19}$ namely transentorhinal cortex, anterior cingulate cortex, amygdala, and insular cortex. ${ }^{12,20}$ For Lewy body variant of Alzheimer's disease (LBVAD), both sets of the above criteria had to be met. Ten patients with pure $\mathrm{AD}, 7$ with LBVAD, and 4 with pure diffuse Lewy body disease (DLBD) had participated in glucose metabolic PET imaging research antemortem at the Division of $\mathrm{Nu}-$ clear Medicine, The University of Michigan. At the time of PET scan, all pure $\mathrm{AD}$ patients had a clinical diagnosis of probable $\mathrm{AD}$ based on NINCDS/ADRDA criteria. ${ }^{21}$ Among LBVAD patients, 4 patients carried a clinical diagnosis of probable $\mathrm{AD}, 2$ patients had probable $\mathrm{AD}$ with extrapyramidal signs, and 1 patient had Parkinson's disease with dementia. Among DLBD patients, 1 patient had a clinical diagnosis of probable $\mathrm{AD}, 1$ patient had probable $\mathrm{AD}$ with extrapyramidal signs, and 2 patients had Parkinson's disease with dementia. A mean Mini-Mental State Examination (MMSE) score $^{22}$ and clinical dementia rating $(\mathrm{CDR})^{23}$ were measured at the time of PET imaging (Table 1). Differences in means for age of onset, age, MMSE score, and CDR at the time of PET scan, and interval between PET and autopsy among $\mathrm{AD}, \mathrm{LBVAD}$, and DLBD were not significant (Analysis of Variance, $p>0.05$ ). Ten age-similar normal controls (6 females, mean age $68 \pm 6$ years and mean MMSE scores of $29 \pm 1$ ), free from neurological abnormalities on the day of PET scan, were included for comparison.

In the second part of the study, cerebral glucose metabolic
PET image sets of 66 probable $\mathrm{AD}$ patients who were recruited between 1989 and 1992 from the Cognitive Disorders Clinic, The University of Michigan, for ongoing research protocols were analyzed retrospectively. All patients had a clinical diagnosis of probable $\mathrm{AD}$ at the time of PET scan. Eleven patients who later had autopsy confirmation of the diagnosis were included in the first part of the study and were excluded from this analysis. One patient showed mild extrapyramidal symptoms, and one patient had experienced possible visual hallucination at the time of the recruitment to PET studies. Exclusion of these patients resulted in 53 patients (see Table 1). Clinical followup occurred at 5 to 13 month intervals, and neurological and neuropsychological evaluations were repeated. Using their clinically documented medical information, these patients were reclassified retrospectively to probable DLB, possible DLB, or remained probable $\mathrm{AD}$ based on recently proposed clinical diagnostic criteria for DLB. ${ }^{6}$ Among the 53 patients, 4 and 9 patients were classified as probable and possible DLB, respectively, and 40 patients remained classified as probable AD. Differences in means for age of onset, age, mean MMSE score, and CDR at the time of PET scan among probable DLB, possible DLB, and probable AD were not significant (Analysis of Variance, $p>0.05$ ).

\section{PET Cerebral Glucose Metabolic Measurement}

Glucose metabolic PET images were obtained using a Siemens ECAT scanner (model 931/08-12, CTI, Knoxville, $\mathrm{TN}$ ) starting at 30 minutes following intravenous injection of $10 \mathrm{mCi}(370 \mathrm{MBq})$ of $\left[{ }^{18} \mathrm{~F}\right]$-2-fluoro-2-deoxy-D-glucose in a dimly lit room with patient's eyes open. ${ }^{24}$ The protocol was approved by the Institutional Review Board of the University of Michigan Medical School.

\section{Data Analysis}

PET image analysis was performed using automated methods to minimize observer biases and to improve reproducibility of results. ${ }^{25,26}$ Briefly, each reconstructed image set was warped to the common stereotactic coordinate system, ${ }^{27,28}$ and gray matter activities were extracted to predefined surface pixels using a three-dimensional stereotactic surface pro-

Table 1. Demographic Data of Patients with Alzheimer's Disease and Dementia with Lewy Bodies

\begin{tabular}{|c|c|c|c|c|c|c|}
\hline Patients & Sex & $\begin{array}{c}\text { Age of Onset } \\
(y r)\end{array}$ & $\begin{array}{c}\text { Age at PET } \\
(\mathrm{yr})\end{array}$ & MMSE $^{a}$ & $\mathrm{CDR}^{\mathrm{a}}$ & $\begin{array}{l}\text { Interval between } \\
\text { PET and Autopsy } \\
\text { (yr) }\end{array}$ \\
\hline \multicolumn{7}{|l|}{ Autopsy-confirmed } \\
\hline Alzheimer's disease & $9 / 1$ & $66 \pm 7$ & $69 \pm 6$ & $14 \pm 6$ & $1.7 \pm 1.0$ & $3.1 \pm 1.0$ \\
\hline $\begin{array}{l}\text { Lewy body variant of Alzheimer's } \\
\text { disease }\end{array}$ & $3 / 4$ & $67 \pm 7$ & $72 \pm 6$ & $11 \pm 7$ & $2.0 \pm 1.0$ & $3.2 \pm 2.0$ \\
\hline $\begin{array}{l}\text { Pure diffuse Lewy body disease } \\
\text { Clinically diagnosed }\end{array}$ & $3 / 1$ & $70 \pm 6$ & $71 \pm 8$ & $18 \pm 13$ & $1.7 \pm 1.2$ & $3.4 \pm 2.6$ \\
\hline $\begin{array}{l}\text { Clinically diagnosed } \\
\text { Probable Alzheimer's disease }\end{array}$ & $16 / 24$ & $65 \pm 8$ & $69 \pm 8$ & $16 \pm 6$ & $1.4 \pm 0.7$ & - \\
\hline Probable dementia with Lewy bodies & $3 / 1$ & $58 \pm 7$ & $66 \pm 3$ & $11 \pm 6$ & $1.5 \pm 1.0$ & - \\
\hline Possible dementia with Lewy bodies & $4 / 5$ & $61 \pm 9$ & $65 \pm 8$ & $17 \pm 5$ & $1.1 \pm 0.4$ & - \\
\hline
\end{tabular}

${ }^{a}$ MMSE and CDR were measured at the time of PET imaging. Mean \pm standard deviation.

$\mathrm{M} / \mathrm{F}=$ male and female subjects; PET $=$ position emission tomography; MMSE $=$ Mini-Mental State Examination scores $^{22} ; \mathrm{CDR}^{2}$ clinical $^{2}$ dementia rating. ${ }^{23}$ 
jection technique (3D-SSP). ${ }^{25,26}$ To increase the sensitivity of the analysis and to obviate invasive arterial blood sampling, data sets were normalized to the pontine activity in which glucose metabolic activity is known to be preserved relatively in $\mathrm{AD}^{17}$ and DLB. ${ }^{12,13}$

Nine cortical regions of interest (ROI) were predefined for each hemisphere based on the stereotactic brain atlas ${ }^{27}$ as follows: lateral parietal association (including Brodmann's cortical areas, BA 5, 7, 39, and 40); lateral temporal association (BA 21, 22, 37, and 38); lateral frontal association (BA 6, $8-11$, and 44-47); lateral occipital association (BA 18 and 19); mesiotemporal (BA 27, 28, and 34); posterior cingulate (BA 23 and 31); anterior cingulate (BA 24 and 32); primary visual (BA 17); and primary sensorimotor (BA 1-4) cortices. In addition, three subcortical ROIs were predefined in the thalamus, striatum, and cerebellar hemispheres. Metabolic activities were averaged between hemispheres, but metabolic asymmetry was examined also in the occipital cortices by an asymmetry index of (right - left)/(right + left).

The Analysis of Variance followed by post-hoc univariate $\mathrm{F}$ tests and Bonferroni correction for multiple comparisons were performed to examine group differences between normal controls versus AD, LBVAD, and DLBD as well as between $\mathrm{AD}$ versus $\mathrm{LBVAD}$ and DLBD. Receiver-operating characteristics (ROC) analysis ${ }^{29}$ was performed to examine the discriminatory accuracy of glucose metabolic PET imaging between DLB and AD. Correlation between regional metabolic reductions and MMSE scores was also examined in DLB patients, and the statistical significance was estimated with Bonferroni multiple comparison adjustment.

Pixel-by-pixel comparisons of metabolic activities were performed to elucidate the metabolic patterns of $\mathrm{AD}$ and DLB over the entire brain. Two-sample $t$ statistic values (converted to $\mathrm{Z}$ ) were calculated between $\mathrm{AD}$ and DLB at each pixel, and the extent and significance of metabolic reduction in each disease category were assessed on the $\mathrm{Z}$ score maps with an approximate statistical $\mathrm{Z}$ threshold of $4.53 .^{30}$

To characterize the nature of regional metabolic reduction further, image-based principal components analysis ${ }^{31,32}$ was applied on the autopsy-confirmed, DLB (LBVAD + DLBD) data. This analysis revealed intercorrelated patterns of regional metabolic changes occurring in DLB brains.

Retrospective analysis of 53 probable $\mathrm{AD}$ patients compared the frequency of metabolic reduction in the primary visual cortex between patients with clinically-diagnosed DLB and probable $\mathrm{AD}$ ( $\mathrm{\kappa}^{2}$ test) using $\mathrm{Z}$-scores defined as (normal mean - patient value)/(normal standard deviation). We also examined the time interval between PET findings and onset of DLB symptoms.

\section{Results}

Autopsy-confirmed AD patients had significant metabolic reductions in the lateral parietal, temporal, and frontal association cortices and posterior cingulate cortex (Table 2, A-N). Metabolic reductions in the primary visual and sensorimotor cortices as well as subcortical structures were relatively mild and were not significant. The region-specific pattern of metabolic reduction in autopsy-confirmed AD was clearly demonstrated on a statistical map (Figure 1, AD). These find-

Table 2. Energy Metabolism in Alzheimer's Disease, Lewy Body Variant of Alzheimer's Disease, and Pure Diffuse Lewy Body Disease ${ }^{\text {a }}$

\begin{tabular}{|c|c|c|c|c|c|c|c|c|c|}
\hline & \multicolumn{4}{|c|}{ Normalized Metabolic Activity (mean $\pm S D$ ) } & \multirow{2}{*}{\multicolumn{5}{|c|}{ Percent Reduction }} \\
\hline & \multirow{2}{*}{$\begin{array}{c}\mathrm{N} \\
(\mathrm{n}=10)\end{array}$} & \multirow{2}{*}{$\begin{array}{c}\mathrm{AD}(\mathrm{A}) \\
(\mathrm{n}=10)\end{array}$} & \multirow{2}{*}{$\begin{array}{l}\text { LBVAD(L) } \\
(\mathrm{n}=7)\end{array}$} & \multirow{2}{*}{$\begin{array}{c}\mathrm{DLBD}(\mathrm{D}) \\
(\mathrm{n}=4)\end{array}$} & & & & & \\
\hline & & & & & $\mathrm{A}-\mathrm{N}^{\mathrm{b}}$ & $\mathrm{L}-\mathrm{N}^{\mathrm{c}}$ & $\mathrm{D}-\mathrm{N}^{\mathrm{d}}$ & $\mathrm{L}-\mathrm{A}^{\mathrm{e}}$ & $D-A^{f}$ \\
\hline \multicolumn{10}{|l|}{ Association cortex } \\
\hline Frontal & $1.40 \pm 0.14$ & $1.12 \pm 0.10$ & $1.11 \pm 0.18$ & $1.14 \pm 0.23$ & $-20 \% \mathrm{~g}$ & $-21 \% \mathrm{~g}$ & $-19 \%$ & $-1 \%$ & $2 \%$ \\
\hline Parietal & $1.35 \pm 0.13$ & $0.90 \pm 0.09$ & $0.88 \pm 0.15$ & $0.94 \pm 0.16$ & $-33 \%^{\mathrm{h}}$ & $-35 \%{ }^{\mathrm{h}}$ & $-30 \%^{\mathrm{h}}$ & $-2 \%$ & $5 \%$ \\
\hline Temporal & $1.25 \pm 0.09$ & $0.91 \pm 0.05$ & $0.94 \pm 0.09$ & $1.02 \pm 0.15$ & $-27 \%^{\mathrm{h}}$ & $-25 \%^{\mathrm{h}}$ & $-18 \%^{\mathrm{g}}$ & $3 \%$ & $13 \%$ \\
\hline Occipital & $1.31 \pm 0.08$ & $1.10 \pm 0.15$ & $0.97 \pm 0.15$ & $0.95 \pm 0.11$ & $-16 \%$ & $-26 \%^{\mathrm{h}}$ & $-28 \%{ }^{\mathrm{h}}$ & $-12 \%$ & $-13 \%$ \\
\hline \multicolumn{10}{|l|}{ Limbic cortex } \\
\hline Posterior cingulate & $1.41 \pm 0.13$ & $1.08 \pm 0.13$ & $1.00 \pm 0.16$ & $1.03 \pm 0.19$ & $-24 \%^{\mathrm{h}}$ & $-29 \%^{\mathrm{h}}$ & $-27 \%{ }^{\mathrm{h}}$ & $-7 \%$ & $-4 \%$ \\
\hline Anterior cingulate & $1.20 \pm 0.11$ & $1.00 \pm 0.11$ & $1.02 \pm 0.13$ & $1.02 \pm 0.17$ & $-17 \%$ & $-15 \%$ & $-15 \%$ & $3 \%$ & $3 \%$ \\
\hline Mesiotemporal & $0.97 \pm 0.06$ & $0.89 \pm 0.06$ & $0.86 \pm 0.07$ & $0.92 \pm 0.08$ & $-8 \%$ & $-11 \%$ & $-5 \%$ & $-3 \%$ & $4 \%$ \\
\hline \multicolumn{10}{|l|}{ Primary cortex } \\
\hline Sensorimotor & $1.47 \pm 0.15$ & $1.28 \pm 0.12$ & $1.29 \pm 0.14$ & $1.27 \pm 0.22$ & $-13 \%$ & $-12 \%$ & $-14 \%$ & $1 \%$ & $-1 \%$ \\
\hline Visual & $1.49 \pm 0.09$ & $1.37 \pm 0.13$ & $1.14 \pm 0.13$ & $1.05 \pm 0.16$ & $-8 \%$ & $-23 \%^{\mathrm{h}}$ & $-29 \%^{\mathrm{h}}$ & $-17 \% \mathrm{~g}$ & $-23 \%^{\mathrm{g}}$ \\
\hline \multicolumn{10}{|l|}{ Subcortex } \\
\hline Thalamus & $1.63 \pm 0.15$ & $1.47 \pm 0.09$ & $1.45 \pm 0.14$ & $1.43 \pm 0.22$ & $-10 \%$ & $-11 \%$ & $-12 \%$ & $-1 \%$ & $-3 \%$ \\
\hline Striatum & $1.47 \pm 0.16$ & $1.36 \pm 0.11$ & $1.33 \pm 0.15$ & $1.45 \pm 0.19$ & $-7 \%$ & $-9 \%$ & $-1 \%$ & $-2 \%$ & $7 \%$ \\
\hline Cerebellum & $1.24 \pm 0.03$ & $1.16 \pm 0.05$ & $1.14 \pm 0.09$ & $1.22 \pm 0.15$ & $-6 \%$ & $-8 \%$ & $-2 \%$ & $-2 \%$ & $4 \%$ \\
\hline \multicolumn{10}{|c|}{$\begin{array}{l}{ }^{a} \text { Posthoc univariate } \mathrm{F} \text { test with a contrast between groups. } \\
\mathrm{b}(\mathrm{A}-\mathrm{N}) / \mathrm{N} \times 100 \text {. } \\
\mathrm{c}(\mathrm{L}-\mathrm{N}) / \mathrm{N} \times 100 \text {. } \\
\mathrm{d}(\mathrm{D}-\mathrm{N}) / \mathrm{N} \times 100 \\
\mathrm{e}(\mathrm{L}-\mathrm{A}) / \mathrm{A} \times 100 \\
\mathrm{f}(\mathrm{D}-\mathrm{A}) / \mathrm{A} \times 100 \text {. } \\
\mathrm{g}_{p}<0.05 \text { (adjusted for multiple comparisons). } \\
{ }^{\mathrm{h}} p<0.005 \text { (adjusted for multiple comparisons). }\end{array}$} \\
\hline $\mathrm{N}=$ normal; $\mathrm{AD}(\mathrm{A})$ & $=$ pure Alzhein & er's disease; LB & $\operatorname{VAD}(\mathrm{I}$ & & & (D) $=$ & & & \\
\hline
\end{tabular}


ings are consistent with previous imaging studies of clinically-diagnosed AD.

DLB patients showed significant metabolic reductions in the lateral association and posterior cingulate cortices, similar to $\mathrm{AD}$, but distinct in the significant reduction found in the occipital lobe particularly primary visual cortex (see Table 2, L-N and D-N). Both LBVAD and DLBD patients showed severe metabolic reduction in the occipital lobe. Metabolic reduction in the visual cortex in LBVAD and DLBD was significantly greater than that in $\mathrm{AD}$ (see Table 2, L-A and D-A). Statistical maps confirmed the marked similarity of metabolic reductions in the lateral parietotemporal and frontal association cortices between $\mathrm{AD}$ and DLB (both LBVAD and DLBD), but distinct differences in the lateral occipital and primary visual cortices (see Fig 1, DLB, LBVAD, and DLBD). Individual analysis of hemispheric averaged metabolic activities in the primary visual cortex showed 8 of 11 patients with DLB had a metabolic reduction greater than the most severe reduction found in $\mathrm{AD}$ patients (Fig 2).

There was a mild metabolic asymmetry in the occipital lobe. Mean asymmetry indices of the occipital lateral cortex were $3 \%$ in $\mathrm{AD}$ (range $0-11 \%$ ), $4 \%$ in DLB $(0-9 \%)$ as compared to $1 \%$ in normal controls $(0-3 \%)$. Metabolic asymmetry in the primary visual cortex was present, but less than that seen in the occipital lateral association cortex (mean 2\%, range $0-5 \%$ in all categories). Based on the lower hemispheric value of metabolic activities in the primary visual cortices, ROC analysis indicated sensitivities and specificities of $90 \%$ and $80 \%$ at $Z=-2.4,87 \%$ and $85 \%$ at $Z=-2.5$, and $83 \%$ and $90 \%$ at $Z=-2.6$, respectively, when discriminating DLB and AD.

Multivariate analysis of regional glucose metabolism in DLB brains revealed that regional metabolic changes did not occur randomly, but in a regionally coordinated manner. Metabolic changes within the occipital lobe including primary visual and association cortices were intercorrelated with each other (Fig 3, PC3, 10\% of metabolic variances in DLB brains explained by this component). However, these changes did not correlate with changes within the anterior component (frontal association cortex-anterior cingulate cortex-caudate nucleus) (see Fig 3, PC1, 49\%) or those within the posterior component (parietotemporal association cortices-posterior cingulate cortex) (see Fig 3, PC2, $24 \%)$. These findings indicate that distinct pathophysiological processes may account for the metabolic abnormalities seen in the occipital lobe of DLB. Correlation analysis in DLB patients demonstrated significant correlation between regional metabolic reductions and MMSE scores in the parietal association cortex $(\mathrm{r}=$ $0.81, p<0.05$ after multiple comparison adjustment), temporal association cortex $(\mathrm{r}=0.81, p<0.05)$, posterior cingulate cortex $(\mathrm{r}=0.75, p<0.05)$, and fron- tal association cortex $(\mathrm{r}=0.75, p<0.05)$. Metabolic reductions in the visual cortex or occipital association cortex, however, did not show a significant correlation $(r=0.32$ and $r=0.58$, respectively, both $p>0.05)$. These findings indicate, again, that the occipital dysfunction in DLB is distinct from the metabolic abnormalities in other association cortices that correlate with dementia severity.

Out of 53 patients with an initial clinical diagnosis of probable AD, 13 patients (25\%) fulfilled the clinical diagnostic criteria for probable or possible DLB during the course of clinical followup. The diagnoses were based on Parkinsonian symptoms in 13 patients, visual hallucination in 4 patients, and cognitive fluctuation in 1 patient. When examining metabolic reduction in the primary visual cortex using $\mathrm{Z}=-2.0$ as a threshold (exceeding this threshold by chance is $2 \%$ ), only 5 of 40 probable $\mathrm{AD}$ patients (13\%) showed metabolic reduction in the visual cortex. In contrast, 5 of 9 possible DLB patients (56\%) and 3 of 4 probable DLB patients (75\%) showed metabolic reduction in the visual cortex. These findings indicated a significantly higher incidence of metabolic reduction in the visual cortex with clinically-diagnosed DLB as compared to probable AD $\left(\kappa^{2}\right.$ test, $\left.p<0.01\right)$. Using a more rigorous discriminative threshold of $Z=-2.5$, estimated from autopsyproven cases as the optimal cutoff (exceeding this threshold by chance is $0.6 \%$ ), 2 of 40 probable AD patients $(5 \%)$ showed metabolic reduction in the primary visual cortex as compared to 2 of 9 possible DLB patients $(22 \%)$ and 3 of 4 probable DLB patients (75\%). Again, the incidence of metabolic reduction in the primary visual cortex with DLB was significantly greater than that in $\mathrm{AD}\left(\kappa^{2}\right.$ test, $\left.p<0.01\right)$. Despite known limitations in accuracy of retrospective clinical diagnosis for DLB, the above cutoff thresholds yielded specificity $88 \%$ and sensitivity $62 \%(Z=-2.0)$ and specificity $95 \%$ and sensitivity $38 \%(Z=-2.5)$, respectively, for the discrimination of clinically diagnosed $\mathrm{AD}$ versus possible and probable DLB.

All 53 patients were recruited initially as probable $\mathrm{AD}$ without extrapyramidal signs, visual hallucination, or cognitive fluctuation, some of whom later developed additional DLB symptoms during the course of the disease. Among clinically-reclassified DLB patients showing metabolic reduction in the primary visual cortex $(Z$ threshold -2.0$)$, mean duration between PET imaging performed at the time of the diagnosis of 'probable AD' and onset of DLB symptoms were 41 months for Parkinsonism (21-78 months), 29 months for visual hallucination (6-50 months), and 51 months for cognitive fluctuation.

\section{Discussion}

This study revealed comparable degrees of metabolic reduction in the parietotemporal and frontal associa- 


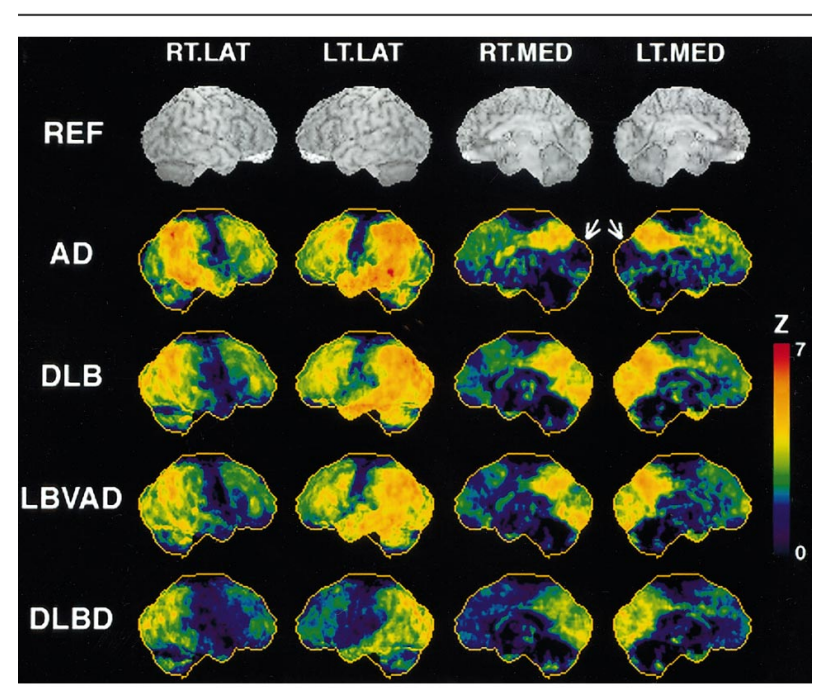

Fig 1. Cerebral metabolic reduction in autopsy-confirmed Alzheimer's disease (AD), dementia with Lewy bodies (DLB), Lewy body variant of Alzheimer's disease (LBVAD), and pure diffuse Lewy body disease (DLBD). DLB represents pooled data of $L B V A D$ and $D L B D$. Color coding represents statistical significance (Z-score) of regional metabolic reduction in comparison to age-similar normal controls. The right lateral (RT.LAT), left lateral (LT.LAT), right medial (RT.MED), and left medial (LT.MED) views of the brain are presented (see reference anatomic images, REF). Red represents more significant metabolic reduction. In $A D$ and $D L B$, there is significant metabolic reduction in the lateral association cortices, but sparing the primary sensorimotor cortex in between frontal and parietal association cortices (RT.LAT and LT.MED). On the medial views of the brain (RT.MED and LT.MED), there is sparing of glucose metabolism in the occipital cortex in $A D$ (arrows), but significant reduction is seen in $D L B, \angle B V A D$, and $D L B D$.

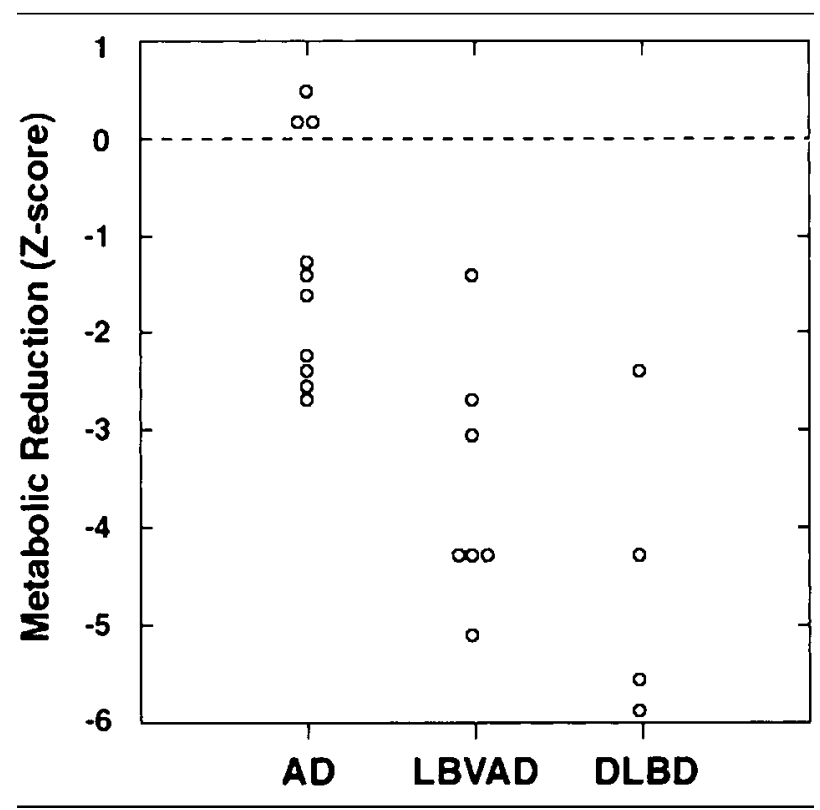

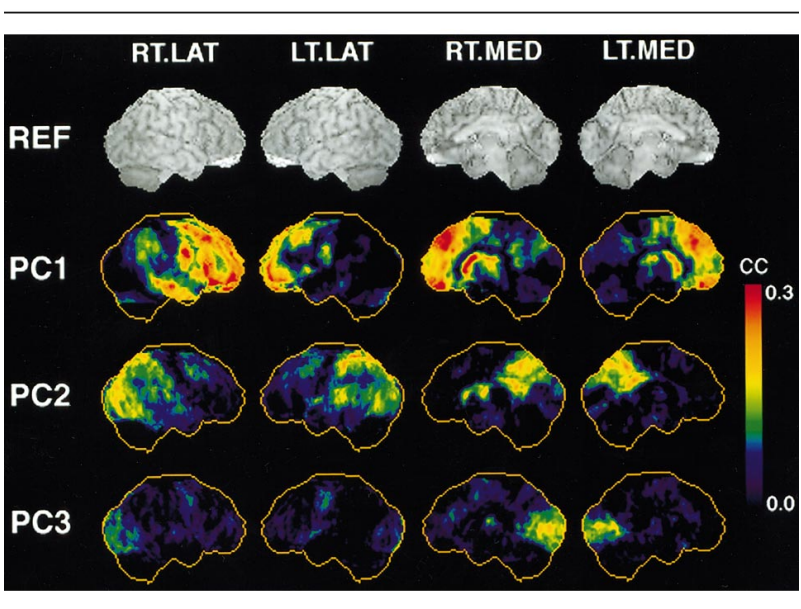

Fig 3. Intercorrelation of regional metabolic changes in $D L B$ as revealed by multivariate correlational analysis. The same views of the brain as Figure 1 are shown. Color coding represents correlation coefficients with latent components (PC1 to PC3). The first component, PC1 represents intercorrelated metabolic changes within the anterior component (frontal association cortex-anterior cingualte cortex-caudate nucleus).

The second component PC2 represents the posterior component (parietotemporal association cortices-posterior cingulate cortex). Occipital changes, PC3, are distinct from these two components, indicating the involvement of a distinct system in the pathophysiology of occipital metabolic abnormalities in DLB.

tion cortices in both autopsy-confirmed DLB and AD, but only DLB showed severe metabolic reductions in the occipital lobe. These findings were similar for both LBVAD and DLBD. Occipital metabolic changes, however, did not correlate with parietotemporal changes in DLB, suggesting impairment of distinct neuronal systems. Retrospective analysis of clinicallydiagnosed probable $\mathrm{AD}$ patients showed that $25 \%$ fulfilled consensus criteria for probable or possible DLB in their clinical courses. These patients had a significantly higher incidence of metabolic reduction in the occipital lobe that preceded the occurrence of some DLB symptoms. These two separate analyses indicate clearly that metabolic reduction in the occipital lobe, particularly the primary visual cortex, is a metabolic signature of DLB. Investigations of distinct metabolic patterns between AD, LBVAD, and DLBD are possible only by means of postmortem confirmation of the diagnoses since there currently is no perfect clinical criteria to distinguish these interrelated diseases. ${ }^{33,34}$

Fig 2. Metabolic reduction in the primary visual cortex in individual patients of autopsy-confirmed $A D, L B V A D$, and DLBD. Metabolic activities of right and left hemispheres are averaged prior to the analysis. Metabolic reduction is expressed as $Z$-scores in comparison to age-similar normal controls. $\angle B V A D$ and $D L B D$ show greater metabolic reduction in the primary visual cortex as compared to $A D$. 
Occipital metabolic (or coupled bloodflow) reduction in PD with dementia has been observed previously, ${ }^{35,36}$ but not discussed critically. Recent investigations, employing sophisticated image analysis, consistently indicated occipital metabolic reductions, in addition to extensive parietotemporal and frontal association metabolic abnormalities, in clinically-diagnosed Parkinson's disease with dementia, clinically-diagnosed DLB, and autopsy-confirmed DLB. ${ }^{12-15,37,38}$ Given that a substantial population of Parkinson's disease with dementia is reported to have cortical Lewy bodies, ${ }^{39}$ occipital metabolic reduction appears to be a biological marker found consistently in patients with Lewy body disease.

What pathophysiology could account for the occipital metabolic reduction in DLB? In DLBD, the density of Lewy bodies was reported to be the lowest in the occipital cortex. ${ }^{19,40}$ Although precise comparisons between antemortem metabolic changes and postmortem pathological findings are difficult due to the time interval, the expression of Lewy bodies in DLB brains does not appear to correlate with the distribution of metabolic changes. This discordance between metabolic and classical pathologic changes, however, is not unique to DLB. ${ }^{41}$ In $\mathrm{AD}$, classical pathologic changes occur initially in the transentorhinal cortex. ${ }^{42}$ In contrast, early metabolic changes occur in the posterior cingulate cortex and lateral association cortices, ${ }^{24}$ which correlate more closely with synaptic alterations revealed by immunocytochemical analysis. ${ }^{43}$ Multivariate analysis showed that occipital metabolic abnormalities were not merely an extension of parietotemporal pathology (see Fig 2), indicating an impairment of distinct neuronal systems. A similar but milder metabolic reduction in the occipital lobe was observed also in Parkinson's disease without dementia. ${ }^{44-46}$ This may or may not indicate preclinical evidence of Lewy body dementia. However, our previous study demonstrated that occipital reduction correlated with nigrostriatal dopaminergic functions, ${ }^{46}$ and indicated a possible pathophysiological relationship in impaired saccade and visual attentional systems. ${ }^{47}$ Metabolic reduction in the visual cortex also coincides clinically with a high prevalence of visual hallucinations in DLB. ${ }^{6}$ Abnormalities in primary visual processing as evidenced by metabolic reduction in DLB and PD, may cause a 'release' of higher visual association cortices and result in visual hallucinations. ${ }^{48}$ Alternatively, visual hallucinations might be caused by neurochemical changes outside of the primary visual system. ${ }^{49}$ Further pathophysiological investigation to account for occipital metabolic reduction in DLB is warranted.

One autopsy study showed $36 \%$ of clinically diagnosed and pathologically confirmed AD patients had cortical and subcortical Lewy bodies. ${ }^{50}$ This frequency was similar to our retrospective analysis of probable
$\mathrm{AD}$ patients who also fulfilled the consensus diagnosis of DLB (25\%), although a detailed metabolic analysis of clinically-diagnosed DLB patients suffers from uncertainty of clinical diagnosis and delay until pathologic confirmation. Interestingly, a majority of probable $\mathrm{AD}$ patients who later fulfilled the consensus diagnostic criteria of DLB did not have clinical symptoms of DLB other than dementia at the time of PET imaging. Glucose metabolic abnormalities preceding clinical symptoms are known to occur with Huntington's disease ${ }^{51}$ and AD. ${ }^{24,52}$ Similar phenomena may occur in occipital metabolism of DLB as seen in this study. Further follow up of the patients involved in our retrospective analysis and postmortem diagnosis will confirm the accuracy of metabolic imaging in the early detection of DLB.

Consistent observation of a metabolic reduction in the medial occipital cortex in DLB suggests the use of functional brain imaging as a potential clinical diagnostic aid to differentiate DLB from AD. The sensitivity in discriminating DLB and $\mathrm{AD}$ in the current study was greater than that with clinical diagnostic criteria applied retrospectively to the data from medical charts. ${ }^{33}$ The sensitivity is also greater than a carefully designed prospective clinicopathological correlation study, ${ }^{10}$ but direct comparison is difficult due to different populations of patients involved in these studies. The specificity of the current study was comparable to those based on retrospective clinical diagnoses, but a carefully designed clinical examination for DLB may outperform in specificity. ${ }^{10}$ Diagnostic accuracy can be adjusted based on a cutoff threshold applied, and can be optimized for the desired purpose of the test (such as maximizing specificity by a higher threshold for the confirmatory identification of DLB). It is also important to note that diagnosis using functional brain imaging can be performed in the initial clinical visit without followup for diagnostic purposes. Similar diagnostic information may be also obtained with more widely available perfusion SPECT imaging. ${ }^{14}$ An alternative approach would be to use dopaminergic imaging. Dopaminergic changes in the striatum showed differential rostocaudal distributions between DLB versus PD and varying degrees of severity between DLB and $\mathrm{PD}$ versus $\mathrm{AD} .^{53}$ These findings have a potential for the in vivo imaging differentiation of DLB versus AD. ${ }^{54}$ One significant advantage of glucose metabolic (or perfusion) imaging, however, is the capacity to differentiate other dementing disorders, such as frontotemporal lobe dementia and vascular dementia, ${ }^{55}$ in a single study. Metabolic imaging fulfills many features of a proposed biomarker for $\mathrm{AD} .^{56}$ The accurate antemortem differential diagnosis of interrelated dementing disorders will help select patients who can benefit from drugs specific to diseases and develop drug trials by 
permitting an accurate selection of patients with more uniform pathology.

Supported in part by the National Institutes of Health (RO1NS24896), the Michigan Alzheimer's Disease Research Center (NIH; P50-AG08671), the University of Michigan Claude D. Pepper Older American Independence Center (NIH; P30-AG08808) and the Department of Energy (DE-FG02-87-ER60551).

We thank Donna J. Cross, BSE, for her assistance in preparing this manuscript; Sid Gilman, MD, the director of Michigan Alzheimer's Disease Research Center, for his continuing support; Stanley Berent, $\mathrm{PhD}$, and Bruno Giordani, $\mathrm{PhD}$, for neuropsychological testing; Robert A. Koeppe, PhD, for his assistance in collecting PET data; PET technologists for their skillful performance in image acquisition; and cyclotron operators and chemists for their production of radiopharmaceuticals.

\section{References}

1. Lewy FH. Paralysis agitans: I. Pathologische anatomie. In Lewandowsky M, ed. Handbuch der Neurologie. Berlin: Springer, 1912:920-933.

2. Kosaka K, Oyanagi S, Matsushita M, Hori A. Presenile dementia with Alzheimer-, Pick- and Lewy-body changes. Acta Neuropathol (Berl) 1976;36:221-233.

3. Eggertson DE, Sima AA. Dementia with cerebral Lewy bodies. A mesocortical dopaminergic defect? Arch Neurol 1986;43: $524-527$.

4. Kuzuhara S, Mori H, Izumiyama $\mathrm{N}$, et al. Lewy bodies are ubiquitinated. A light and electron microscopic immunocytochemical study. Acta Neuropathol (Berl) 1988;75:345-353.

5. Lennox G, Lowe J, Morrell K, et al. Anti-ubiquitin immunocytochemistry is more sensitive than conventional techniques in the detection of diffuse Lewy body disease. J Neurol Neurosurg Psychiatry 1989;52:67-71.

6. McKeith LG, Galasko D, Kosaka K, et al. Consensus guidelines for the clinical and pathologic diagnosis of dementia with Lewy bodies (DLB): report of the consortium on DLB international workshop. Neurology 1996;47:1113-1124.

7. Liberini P, Memo M, Spano P. Lewy body pathology and heterogeneity of Alzheimer's disease. JAMA 1995;274:1199.

8. Olichney JM, Galasko D, Salmon DP, et al. Cognitive decline is faster in Lewy body variant than in Alzheimer's disease. Neurology 1998;51:351-357.

9. McKeith I, Fairbairn A, Perry R, et al. Neuroleptic sensitivity in patients with senile dementia of Lewy body type. BMJ 1992; 305:673-678.

10. McKeith IG, Ballard CG, Perry RH, et al. Prospective validation of consensus criteria for the diagnosis of dementia with Lewy bodies. Neurology 2000;54:1050-1058.

11. Case records of the Massachusetts General Hospital. Weekly clinicopathological exercises. Case 7-1998. A 74-year-old man with dementia, Parkinsonism, and an insular lesion. N Engl J Med 1998;338:603-610.

12. Albin RL, Minoshima S, D'Amato CJ, et al. Fluoro-deoxyglucose positron emission tomography in diffuse Lewy body disease. Neurology 1996;47:462-466.

13. Vander Borght T, Minoshima S, Giordani B, et al. Cerebral metabolic differences in Parkinson's and Alzheimer's diseases matched for dementia severity. J Nucl Med 1997;38:797-802.

14. Donnemiller E, Heilmann J, Wenning GK, et al. Brain perfusion scintigraphy with $99 \mathrm{mTc}-\mathrm{HMPAO}$ or $99 \mathrm{mTc}-\mathrm{ECD}$ and 123I-beta-CIT single-photon emission tomography in dementia of the Alzheimer-type and diffuse Lewy body disease. Eur J Nucl Med 1997;24:320-325.
15. Imamura $T$, Ishii $K$, Sasaki $M$, et al. Regional cerebral glucose metabolism in dementia with Lewy bodies and Alzheimer's disease: a comparative study using positron emission tomography. Neurosci Lett 1997;235:49-52.

16. Kuhl DE. Imaging local brain function with emission computed tomography. Radiology 1984;150:625-631.

17. Minoshima S, Frey KA, Foster NL, Kuhl DE. Preserved pontine glucose metabolism in Alzheimer disease: a reference region for functional brain image (PET) analysis. J Comput Assist Tomogr 1995;19:541-547.

18. Khachaturian ZS. Diagnosis of Alzheimer's disease. Arch Neurol 1985;42:1097-1105.

19. Gomez-Tortosa E, Newell K, Irizarry MC, et al. Clinical and quantitative pathologic correlates of dementia with Lewy bodies. Neurology 1999;53:1284-1291.

20. Sima AA, Clark AW, Sternberger NA, Sternberger LA. Lewy body dementia without Alzheimer changes. Can J Neurol Sci 1986;13:490-497.

21. McKhann G, Drachman D, Folstein M, et al. Clinical diagnosis of Alzheimer's disease: report of the NINCDS-ADRDA Work Group under the auspices of Department of Health and Human Services Task Force on Alzheimer's Disease. Neurology 1984;34:939-944.

22. Folstein MF, Folstein SE, McHugh PR. "Mini-mental state.” A practical method for grading the cognitive state of patients for the clinician. J Psychiatr Res 1975;12:189-198.

23. Gelb DJ, St. Laurent RT. Alternative calculation of the global clinical dementia rating. Alzheimer Dis Assoc Disord 1993;7: 202-211.

24. Minoshima S, Giordani B, Berent S, et al. Metabolic reduction in the posterior cingulate cortex in very early Alzheimer's disease. Ann Neurol 1997;42:85-94.

25. Minoshima S, Ficaro EP, Frey KA, et al. Data extraction from brain PET images using three-dimensional stereotactic surface projections. Quantitative functional brain imaging with positron emission tomography. Carson RE, Daube-Witherspoon ME, and Herscovitch P, Eds. San Diego: Academic Press, 1998:133-137.

26. Minoshima S, Frey KA, Koeppe RA, et al. A diagnostic approach in Alzheimer's disease using three-dimensional stereotactic surface projections of fluorine-18-FDG PET. J Nucl Med 1995;36:1238-1248.

27. Talairach J, Tournoux P. Co-planar stereotaxic atlas of the human brain. New York: Thieme, 1988.

28. Minoshima S, Koeppe RA, Frey KA, Kuhl DE. Anatomic standardization: linear scaling and nonlinear warping of functional brain images. J Nucl Med 1994;35:1528-1537.

29. Metz CE. ROC methodology in radiologic imaging. Invest Radiol 1986;21:720-733.

30. Worsley KJ, Marrett S, Neelin P, Evans AC. A unified statistical approach for determining significant signals in location and scale space images of cerebral activation, In: Myers R, Ed. Quantification of brain function using PET. San Diego: Academic Press, 1996:327-333.

31. Minoshima S, Ficaro EP, Koeppe RA, et al. Interpretation of regional neurochemical and anatomic changes in neurodegeneration: multivariate approach. Neuroimage 1998;7:A62.

32. Anzai Y, Minoshima S, Wolf GT, Wahl RL. Head and neck cancer: detection of recurrence with three-dimensional principal components analysis at dynamic FDG PET. Radiology 1999; 212:285-290.

33. Luis CA, Barker WW, Gajaraj K, et al. Sensitivity and specificity of three clinical criteria for dementia with Lewy bodies in an autopsy-verified sample. Int J Geriatr Psychiatry 1999;14: 526-533.

34. Papka M, Rubio A, Schiffer RB, Cox C. Lewy body disease: can we diagnose it? J Neuropsychiatry Clin Neurosci 1998;10: 405-412. 
35. Spampinato U, Habert MO, Mas JL, et al. (99mTc)-HM-PAO SPECT and cognitive impairment in Parkinson's disease: a comparison with dementia of the Alzheimer type. J Neurol Neurosurg Psychiatry 1991;54:787-792.

36. Liu RS, Lin KN, Wang SJ, et al. Cognition and 99TcmHMPAO SPECT in Parkinson's disease. Nucl Med Commun 1992;13:744-748.

37. Ishii K, Imamura T, Sasaki M, et al. Regional cerebral glucose metabolism in dementia with Lewy bodies and Alzheimer's disease. Neurology 1998;51:125-130.

38. Turner RS, Chervin RD, Frey KA, et al. Probable diffuse Lewy body disease presenting as REM sleep behavior disorder. Neurology 1997;49:523-527.

39. Hughes AJ, Daniel SE, Blankson S, Lees AJ. A clinicopathologic study of 100 cases of Parkinson's disease. Arch Neurol 1993;50:140-148.

40. Rezaie P, Cairns NJ, Chadwick A, Lantos PL. Lewy bodies are located preferentially in limbic areas in diffuse Lewy body disease. Neurosci Lett 1996;212:111-114.

41. Jagust WJ. Functional imaging patterns in Alzheimer's disease. Relationships to neurobiology. Ann NY Acad Sci 1996;777: $30-36$.

42. Braak H, Braak E. Neuropathological stageing of Alzheimerrelated changes. Acta Neuropathol (Berl) 1991;82:239-259.

43. Masliah E, Terry RD, Alford M, et al. Cortical and subcortical patterns of synaptophysinlike immunoreactivity in Alzheimer's disease. Am J Pathol 1991;138:235-246.

44. Kuhl DE, Metter EJ, Riege WH. Patterns of local cerebral glucose utilization determined in Parkinson's disease by the [18F]fluorodeoxyglucose method. Ann Neurol 1984;15:419-424.

45. Eberling JL, Richardson BC, Reed BR, et al. Cortical glucose metabolism in Parkinson's disease without dementia. Neurobiol Aging 1994;15:329-335.

46. Bohnen NI, Minoshima S, Giordani B, et al. Motor correlates of occipital glucose hypometabolism in Parkinson's disease without dementia. Neurology 1999;52:541-546.

47. Hikosaka O, Sakamoto M, Miyashita N. Effects of caudate nucleus stimulation on substantia nigra cell activity in monkey. Exp Brain Res 1993;95:457-472.

48. Manford M, Andermann F. Complex visual hallucinations. Clinical and neurobiological insights. Brain 1998;121:1819-1840.

49. Perry EK, McKeith I, Thompson P, et al. Topography, extent, and clinical relevance of neurochemical deficits in dementia of Lewy body type, Parkinson's disease, and Alzheimer's disease. Ann NY Acad Sci 1991;640:197-202.

50. Hansen L, Salmon D, Galasko D, et al. The Lewy body variant of Alzheimer's disease: a clinical and pathologic entity. Neurology 1990;40:1-8.

51. Kuhl DE, Markham CH, Metter EJ, et al. Local cerebral glucose utilization in symptomatic and presymptomatic Huntington's disease. Res Publ Assoc Res Nerv Ment Dis 1985;63:199-209.

52. Reiman EM, Caselli RJ, Yun LS, et al. Preclinical evidence of Alzheimer's disease in persons homozygous for the epsilon 4 allele for apolipoprotein E. N Engl J Med 1996;334:752-758.

53. Piggott MA, Marshall EF, Thomas N, et al. Striatal dopaminergic markers in dementia with Lewy bodies, Alzheimer's and Parkinson's diseases: rostrocaudal distribution. Brain 1999;122: $1449-1468$.

54. Walker Z, Costa DC, Janssen AG, et al. Dementia with lewy bodies: a study of post-synaptic dopaminergic receptors with iodine-123 iodobenzamide single-photon emission tomography. Eur J Nucl Med 1997;24:609-614.

55. Salmon E, Sadzot B, Maquet P, et al. Differential diagnosis of Alzheimer's disease with PET. J Nucl Med 1994;35:391-398.

56. Thies B, Truschke E, Morrison-Bogorad M, Hodes RJ. Consensus report of the Working Group on: molecular and biochemical markers of Alzheimer's disease. Neurobiol Aging 1999;20:247. 